\title{
Hemorragia suprarrenal en el recién nacido
}

\author{
Jaime Burgos M. ${ }^{1}$; Sergio Cassis $C^{2}$; Gabriela Kunstmann $Z^{1-3}$; \\ Américo Hernández L. ${ }^{1}$
}

\section{Adrenal haemorrhage in newborn infants}

\begin{abstract}
A retrospective clinical description of 7 newborn infants with adrenal haemorchage. Alf patients were born at term by vaginal deliverv. Their birth weights ranged between 2.550 and 3.950 , and onlv one was smali for gestational age. Two patierits had Apgar scores less than 6 at one rrinute and both were born by forceps delivery. The first symptor of adrenal haemorhage was an abdominal mass. Diagnosis was confirmed bv u:trasonography demostrat. ing a ight adrenal ecogenic mass wich dispiaced the Kidney which disappeared along a few and all patients survived. (Key words: newborn infants, adrenal haemorhage, echography, diagnosis.)
\end{abstract}

La hemorragia suprarrenal (HSR) en el recién nacido (RN) es infrecuente'. No encontramos referencias de la incidencia clinica exacta, sin embargo, existen estudios anatomopatológicos que las han detectado en $6 \%$ de las autopsias realizadas en $\mathrm{RN}^{2}$.

En los RN pueden ocurrir condiciones patológicas que, ocasionalmente, se asocian a HSR, entre las cuales destaçan los partos traumáticos, asfixia perinatal. septicemia y trastornos de coagulación $n^{3,4}$.

Las manifestaciones clínicas dependen de la magnitud de la hemorragia $y$ el compromiso funcional de la glandula. En general se evidencian por el hallazgo de una masa abdominal de flanco, ictericia, anemia, con o sin compromiso hemo. dinámico $^{1,4,5}$; excepcionalmente existe insuficiencia suprartenal aguda. Otras son asintomáticas y pueden aparecer, tardiamente, como imágenes calcificadas en estudios radiológicos posteriores. La pielografía y la ecografía son los elementos de laboratorio fundamentales en el diagnóstico y seguimiento de la HSR.

En la mayoría de las HSR se puede anticipar un pronóstico benigno, aunque ocurren casos fatales, asociados a hemorragias extensas y bilaterales.

1. Lnidad de Neonatología, Servicio de Pediatría Hospital San Juan de Dios.

2. Becado Pediatría, Facultad de Medicina, División Occidente, Lniversidad de Chile.

3. Departamento de Pedjatría, Facultad de Medicina, División Occidente, Universidad de Chile.
Esta presentación tiene como propósito recordar las principales manifestaciones clínicas y subrayar lá contribución de la ultrasonografía en la identificación de dicha entidad.

\section{Material y Método}

Se describen retrospectivamente las características clínicas y de laboratorio, incluyendo los resultados de la ultrasonografía renal de 7 casos de hemorragia suprarrenal en $\mathrm{RN}$, que fueron confirmados entre los ninios nacidos en el servicio de obstetricia del Hospital San Juan de Dios de Santiago, Chile, durante los años 1983 a 1989. En dicho período el número de partos anuales fue de 7.500 .

\section{Resultados}

Los siete casos de HSR observados correspondieron a RN de térnino con edades gestacionales entre $381 / 2$ y 40 semanas. Todos ellos con peso de nacimiento adecuado a su edad gestacional.

Todos los niños nacieron por parto vaginal y en tres RN hubo dificultad durante el periodo expulsivo del parto, lo que obligó z algún tipo de maniobra obstétrica.

Sólo en dos RN la calificación de Apgar al minuto fue igual o inferior a 6 (tabla 1 ).

Los siete casos de HSR se diagnosticaron dentro de los cinco días de vida y en todos ellos se encontró una masa abdominal ubicada en flanco derecho, que constituyó el hallazgo clínico inicial de sospecha diagnóstica. No encontramos ningún caso de HSR bilateral en el período 
Tabla 1

Antecedentes perinatales y hallazgos físicos relevantes

\begin{tabular}{|c|c|c|c|c|}
\hline Paciente & Sexo & $\begin{array}{l}\text { Edad gestacional } \\
\text { Peso nacimiento }\end{array}$ & $\begin{array}{l}\text { Apgat } \\
1 \text { y } 5 \text { min }\end{array}$ & $\begin{array}{l}\text { Vía de parto y } \\
\text { presentación }\end{array}$ \\
\hline 1 & 5 & $\begin{array}{l}381 / 2 \text { semanas } \\
3,20 \mathrm{~kg}\end{array}$ & 9 y 10 & $\mathbf{E} \cdot \mathbf{C}$ \\
\hline 2 & $\mathbf{M}$ & $\begin{array}{l}391 / 2 \text { semanas } \\
3,61 \mathrm{~kg}\end{array}$ & 9 y 9 & $\mathrm{E}-\mathrm{C}$ \\
\hline 3 & $\mathbf{M}$ & $\begin{array}{l}40 \text { semanas } \\
3,95 \mathrm{~kg}\end{array}$ & 9 y 9 & $\begin{array}{l}\text { E - C } \\
\text { Expulsivo de1enido }\end{array}$ \\
\hline 4 & $\mathbf{M}$ & $\begin{array}{l}381 / 2 \text { semanas } \\
3,25 \mathrm{~kg}\end{array}$ & $8 y 9$ & $E \cdot C$ \\
\hline 5 & $F$ & $\begin{array}{l}391 / 2 \text { semanas } \\
3,63 \mathrm{~kg}\end{array}$ & 6 y 8 & $\begin{array}{l}\text { fiorceps por expul } \\
\text { sivo detenido } \\
\text { C }\end{array}$ \\
\hline 6 & $\mathbf{M}$ & $\begin{array}{l}40 \text { semanas } \\
3,81 \mathrm{~kg}\end{array}$ & 9 y 9 & $E-C$ \\
\hline 7 & $\mathbf{M}$ & $\begin{array}{l}381 / 2 \text { semanas } \\
2,55 \mathrm{~kg}\end{array}$ & $4 y_{8}$ & $\begin{array}{l}\text { Fórceps por cesárea } \\
\text { anterior } \\
\text { C }\end{array}$ \\
\hline
\end{tabular}

$F=$ Fenenino

M = Masculino

E $=$ Espontáneo

$C=$ Cefálica

comprendido por este estudio. Todos los recién nacidos, excepto uno, tuvieron hiperbilirrubinemia que obligó, en $\operatorname{los} \operatorname{casos} 1,5$ y 6 , a realjzar exanguineotransfusión.

La anemia ocurrió en dos de los siete casos, manifestándose en forma precoz y severa en el caso tres y requirió tres transfusiones de sangre. Este paciente evolucionó desde la segunda semana con cifras elevadas de presión arterial sistôlica que fluctuaron entre 100 y $130 \mathrm{mmHg}$; el estudio de renina plasmatica demostró una elevación a $36 \mu \mathrm{g} / \mathrm{ml} \cdot \mathrm{h}$. Este nif̂o se trató con propranolol durante tres meses, con normalización de las cifras tensionales para su edad.

En ninguno de estos niños se detectaron signos clínicos de insuficiencia suprarrenal aguda ni se estudió la función suprarrenal. La función renal fue normal en todos ellos.

En la ecografía abdominal destacó el hallazgo de una masa hiperecogénica en la zona suprartenal que desplazaba al riñon en dirección caudal y que en los controles posteriores varió a una zona libre de ecos con reducción del tamaño y normalización ecográfica entre la tercera y la octava semana. Estos hallazgos ecográficos fueron idénticos en los siete niños (figuras 1, 2 y 3).

Como hallazgos mónidos encontramos una coartación aórtica en el caso 4, que fue operada al mes de vida y una encefalopatíc hipóxico is. quémica, en el caso 7, de evolución benigna (tabla 2).

No hubo letalidad en nuestra serie.

\section{Discusión}

El mecanismo básico por el cual se produce la HSR no está aún aclarado, sin embargo, se identifica asociado a factores predisponentes, tales como: partos traumáticos, asfixia perinatal, septicemia y trastornos de coagulación ${ }^{3}$. Al analizar los antecedentes clinicos en nuestra casuistica es interesante destacar que sólo en el caso 7 estuvo presente, objetivamente, uno de estos factores y correspondió a asfixia perinatal. La explicación de la causa de la HSR en los otros pacientes no es clara, pero, debido a que en los casos 3, 5, 7 hubo dificultad durante el periodo 


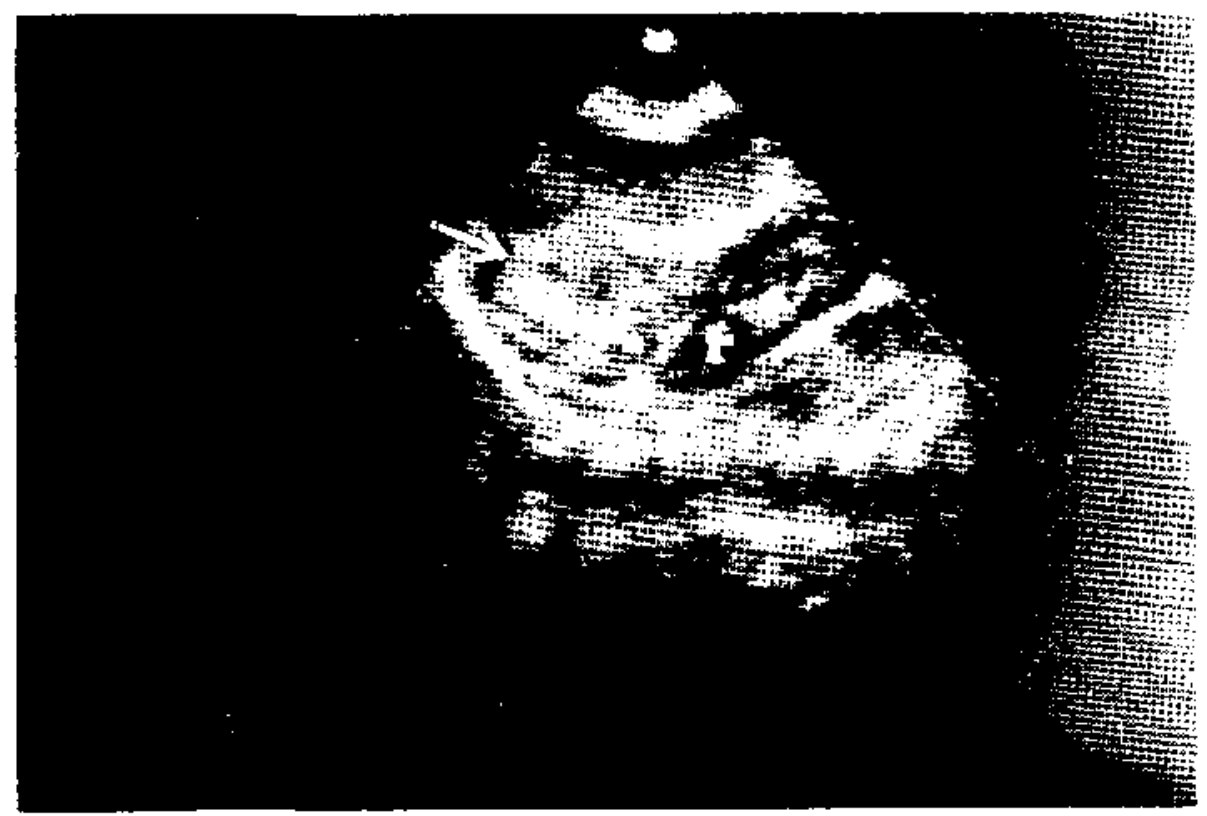

Figura I: Ecografía abdominal; corte longitudinal de hipocondrio derecho. Masa ecogénica homogénea de $3,2 \mathrm{~cm}$ que desplaza al riñón derecho. Caso 3.

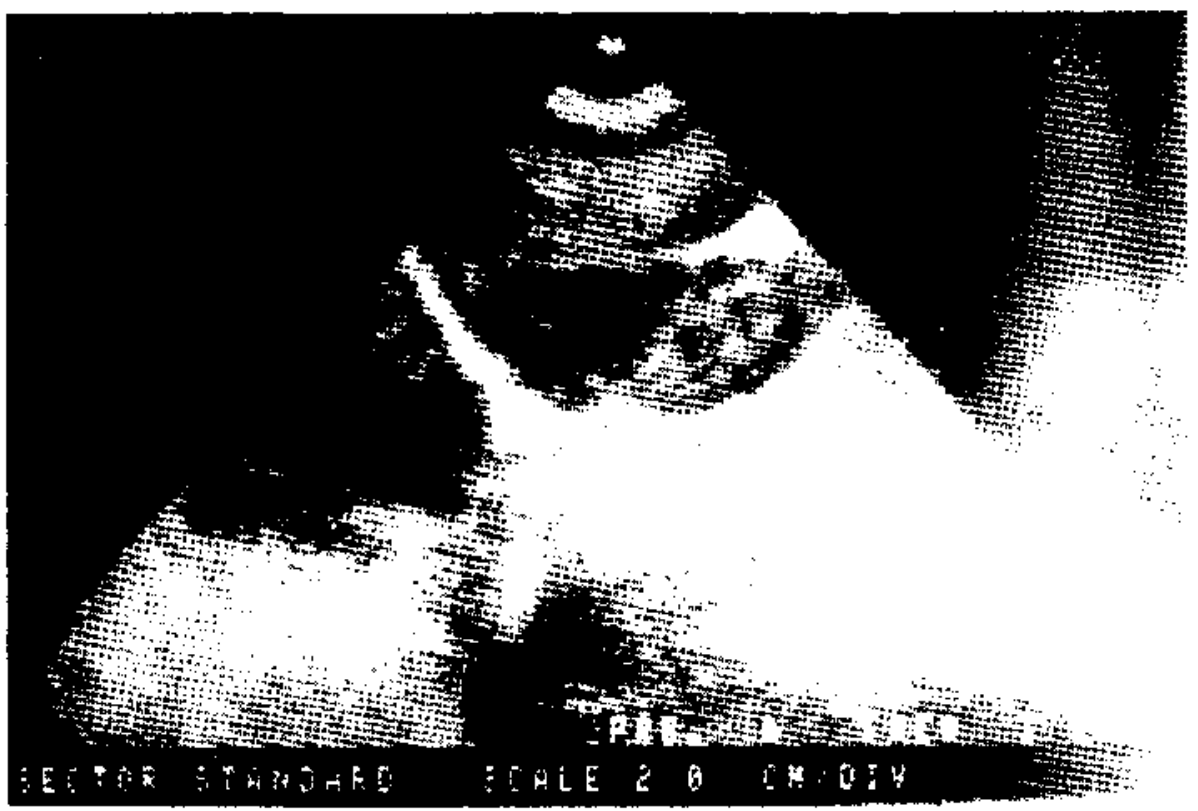

Figura 2: Control a los 15 días. La masa se ha licuado y se observa una estructura hipoecogénica con escasos ecos fuertes en su interior. 


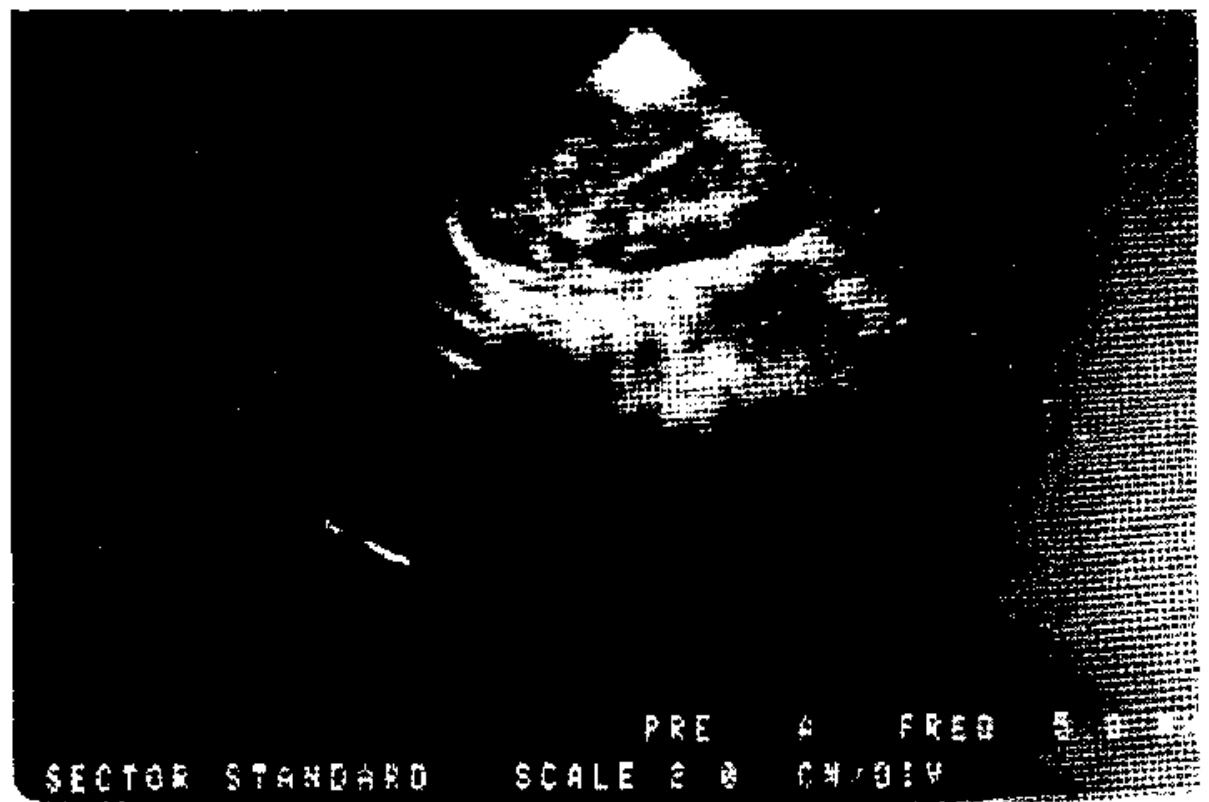

Figura 3: Control a los 2 meses, Regresión de la nasa suprarrenal y riñón en posición normal.

expulsivo del parto, planteamos la posibilidad, sugerida por otros autores ${ }^{1,3,4}$, que durante el trabajo de parto y, o, periodo expulsivo se generó un aumento de la presión intraabdominal fetal con congestión venosa intraglandular y hemorra. gia concomitante. Apoya esta hipótesis la localización derecha de la hemorragia en todos los casos, caracteristica común reportada en distintas publicaciones ${ }^{3,4}$, Jo que demuestra una mayor vulnerabilidad de la glándula suprarrenal derecha, debido a que la vena suprarrenal derecha drena directamente en la vena cava inferior y la expone a cambios de presión, a diferencia de la izquierda, la cual to hace en la vena renal y otras colaterales venosas. Por lo tanto, frente a un aumento de presión de la vena cava inferior por compresión abdominal fetal durante el parto, existe mayor riesgo de hemorragia glandular al lado derecho ${ }^{1,4}$

La ecografía contribuye en forma importante a la detección de la HSR y permite descartar otras causas de masa de flanco ${ }^{3,4,11,12}$. A modo de conclusión, subrayamos el concepto de que hoy en día la ecografía es el método de elección para el estudio y seguimiento de la HSR en el RN.

E1 tratamiento de la HSR $^{1,3,5}$ consiste esencialmente en medidas destinadas a preservar la función cardiovascular y manejar las condiciones patológicas asociadas. En todos nuestros casos el manejo fue conservador y no fue necesario tratamiento quirúrgico, que se plantearía sólo ante la eventualidad de sangramiento incontrolable, no autolimitado ${ }^{1}$ o de una infección del hematoma ${ }^{12}$.

La terapia sustitutiva corticoesteroidal se reserva para situaciones de insuficiencia supra. rrenal aguda y choque, que en general derivan de HSR bilateral extensá.

En el seguimiento posterior al período neonatal sólo uno de los siete casos tuvo complicaciones atribuibles a su HSR, corresponde a la hipertensión arterial presentada por el caso 3 , interpretada como de origen renovascular, como efecto de la compresión del pedículo renal por la masa suprarrenal. Este mecanismo ha sido reconocido en publicaciones anteriores ${ }^{6-10}$.

\section{Resumen}

Se presentan siete casos de hemorragia supra. renal en recién nacidos. Todos nacieron en la maternidad del Hospital San Juan de Dios, su peso de nacimiento varió entre 2.550 y $3.950 \mathrm{~g}$, todos eran de término, nacieron en parto vaginal. En sólo dos la puntuación de Apgar en el primer minuto fue igual o menor a seis. La primera ma- 
Tabla 2

Evolución clínica y ecográfica en 7 RN con HSR

\begin{tabular}{|c|c|c|c|c|}
\hline Paciente & $\begin{array}{l}\text { Edad de } \\
\text { presentación }\end{array}$ & $\begin{array}{l}\text { Síntomas de } \\
\text { presentación y } \\
\text { ubieación HSR }\end{array}$ & $\begin{array}{l}\text { Diagnóstico y } \\
\text { evolución } \\
\text { ecográfica }\end{array}$ & Complicaciones \\
\hline 1 & $2^{\circ}$ día & $\begin{array}{l}\text { Masa flanco derecho } \\
\text { hiperbjlir rubinemia } \\
20 \mathrm{mg} / \mathrm{dl}\end{array}$ & $\begin{array}{l}+ \\
(-) \text { a la } 4 a \\
\text { semana }\end{array}$ & - \\
\hline 2 & $2^{\circ}$ dia & $\begin{array}{l}\text { Masa flanco derecho } \\
\text { hiperbjlirrubjinemia } \\
16.6 \mathrm{mg} / \mathrm{dl}\end{array}$ & $\begin{array}{l}(+) \\
(-) \text { a la } 6^{a} \\
\text { semana }\end{array}$ & - \\
\hline 3 & ler día & $\begin{array}{l}\text { Anemia (hto } 28 \% \text { ) } \\
\text { masa flanco } \\
\text { derecho } \\
\text { hiperbilirubinemia } \\
13.7 \mathrm{mg} / \mathrm{dl}\end{array}$ & $\begin{array}{l}(+) \\
(-) \text { a la } 6^{\mathrm{a}} \\
\text { semana }\end{array}$ & $\begin{array}{l}\text { hipertensión } \\
\text { arterial }\end{array}$ \\
\hline 4 & jer día & $\begin{array}{l}\text { Masa flanco } \\
\text { derecho } \\
\text { hiperbilirubinemia } \\
15 \mathrm{mg} / \mathrm{dl}\end{array}$ & $\begin{array}{l}(+) \\
(-) \text { a la } 4^{a}\end{array}$ & - \\
\hline 5 & $1^{\mathrm{er}}$ & $\begin{array}{l}\text { Masa flanco } \\
\text { derecho } \\
\text { hiperbilirrubinemia } \\
20 \mathrm{mg} / \mathrm{dl}\end{array}$ & $\begin{array}{l}(+) \\
(-) \text { a la } 4^{a} \\
\text { semana }\end{array}$ & - \\
\hline 6 & $5^{\circ} \mathrm{d}$ la & $\begin{array}{l}\text { Hliperbilirrubinem ia } \\
25 \mathrm{mg} / \mathrm{d} \text { l } \\
\text { masa tlanco } \\
\text { derecho }\end{array}$ & $\begin{array}{l}(+) \\
(-) \text { a la } 5^{a} \\
\text { semana }\end{array}$ & - \\
\hline 7 & $2^{\circ}$ día & $\begin{array}{l}\text { Anemia (h10. } 30 \% \text { ) } \\
\text { Masa flanco } \\
\text { derecho }\end{array}$ & $\begin{array}{l}(+) \\
(-) \text { a las } 3 \\
\text { sernana }\end{array}$ & - \\
\hline
\end{tabular}

nifestación en cada uno de estos RN fue una masa abdominal del flanco derecho y el diagnóstico se confirmó mediante ecografías abdominales que mostraron invariablemente una masa suprarrenal derecha que desplazaba el riñón en sentido caudal. Las lesiones evolucionaron desapareciendo en el plazo de 6 semanas. Tres pacientes requirieron exanguineotransfusiones por hiperbilirrubinemia, dos tuvieron anemia severa y uno hipertensión arterial que requirió 3 meses de tratamiento con propranolol. Todos sobrevivieron sin secuelas.

(Palabras clave: hemorragia suprarrenal, recién nacido, masa abdominal, ultrasonografía abdominal.)

\section{Agradecimientos}

Los autores desean expresas sus agradecimientos al Dr. Juan Antonio Escaffi J., por la ayuda como colaborador en la preparación y revisión de este trabajo.

\section{Referencias}

1. Black, J.: Williams, D.J.: Natural history of adrenal hemorrhage in the newborn. Arch Dis Cliild 1973; 48: $183-190$.

2. Benveniste, S.; Las Heras, J.; Mirkin, D.; Fuentes, A.: Henorragia suprarrenal en el recién nacido. Rev Chil Pediatr 1974; 45 ; 119-122.

3. Kuri, F.J.; Alton, D.J.: Hardy, B.E.; Cook, G.T. and Churchilt, B.M.: Adrenal Hemorrhage in neonates: report of 5 cases and review of the litera. ture. J Úrol 1980:124: 684-687. 
4. Contu, D,; Abrego, V.; Arce, R.; Elenes, $F_{.}$: Diagnóstico y seguimiento con ultrasonido de un caso de hemorragia suprarrenal neonatal. Bol Med Hosp Infant Méx 1984; 41:112-115.

5. Smith, F.A.; Fr and Middleton, R.G.: Neonatal Adrenal Hemorthage. J Uro1 1979; 122: 674-677.

6. Norero, C.: Hipertensión Arterial en Recién $\mathrm{Na}$ cidas. Rev Chil Pediatr $1986,57: 465-470$.

7. Bensman, A.; Neurenschwander. S.; Lavollay, B.; Gruner, M.: Hypertension Arterialle et compsession du pédicule vasculaire ténal par un hematome de la Surrénale chez un nouveau-ná. Ann Pediatr $1982 ; 29: 670-672$.

8. Saieh, C.: Dioz, V.; Valdés, F,; Ramírez, K.; Ar.

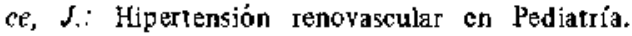
Rey Chil Pediatr 1987; 58: 382-386.
9. Sirote, L.; Stratss, S.; Rechnitz, Y.: Landinan, 1.: Aelitzky. F.: Transient Obstruction of the Kidney and hypertension due to neonatal Adrenal hemorrhage Helv Paediatr Acta 1985; 40: 177181.

10. Parrot, Th.: Woodard, J.: Trulock $T$, and Clenn, J.F.: Segmental renal yein renins and pattial nephectamy for Hipertension in children. J Urol 1984; 131: 736-739.

11. Cohen, E.K.: Daneman, A.; Strunger, D.A.; Soto, $G$; Thower, $P_{, i}$ Focal Adrenal Hemorrhage. A new U.S. apperance. Radiology 1986: 161: 631633.

12. Atkinson, G. Fr.; Kodroff, M.B.: Soy, B. Ir.: Ricketts, R.R.: Adrenal abscess in the nconate. Radiology 1985;155:101-104. 